\title{
ORIGINAL ARTICLE \\ Reduction in the cumulative effect of stress-induced inbreeding depression due to intragenerational purging in Drosophila melanogaster
}

\begin{abstract}
LS Enders ${ }^{1,2}$ and L Nunney ${ }^{1}$
Environmental stress generally exacerbates the harmful effects of inbreeding and it has been proposed that this could be exploited in purging deleterious alleles from threatened inbred populations. However, understanding what factors contribute to variability in the strength of inbreeding depression (ID) observed across adverse environmental conditions remains a challenge. Here, we examined how the nature and timing of stress affects ID and the potential for purging using inbred and outbred Drosophila melanogaster larvae exposed to biotic (larval competition, bacteria infection) and abiotic (ethanol, heat) stressors compared with unstressed controls. ID was measured during (larval survival) and after (male mating success) stress exposure. The level of stress imposed by each stressor was approximately equal, averaging a $42 \%$ reduction in outbred larval survival relative to controls. All stressors induced on average the same ID, causing a threefold increase in lethal equivalents for larval survival relative to controls. However, stress-induced ID in larval success was followed by a 30\% reduction in ID in mating success of surviving males. We propose that this fitness recovery is due to 'intragenerational purging' whereby fitness correlations facilitate stress-induced purging that increases the average fitness of survivors in later life history stages. For biotic stressors, post-stress reductions in ID are consistent with intragenerational purging, whereas for abiotic stressors, there appeared to be an interaction between purging and stress-induced physiological damage. For all stressors, there was no net effect of stress on lifetime ID compared with unstressed controls, undermining the prediction that stress enhances the effectiveness of populationlevel purging across generations.
\end{abstract}

Heredity (2016) 116, 304-313; doi:10.1038/hdy.2015.103; published online 25 November 2015

\section{INTRODUCTION}

Wild populations face a continuous onslaught of natural and anthropogenic stresses including seasonal changes, drought, disease, pollution, habitat loss and competition from invasive species (Loeschcke et al., 2004; Frankham, 2005). When taken to extremes, environmental stress can threaten the survival of a population, but more generally the effect of stress is to significantly reduce the fitness of individuals in a population relative to more benign conditions (Hoffmann and Parsons, 1991). As a result, stress is recognized as having major implications for both short-term survival and long-term adaptation (Loeschcke et al., 2004; Frankham, 2005; Bijlsma and Loeschcke, 2012).

The deleterious effects of stress are often amplified in inbred individuals (Armbruster and Reed, 2005; Fox and Reed, 2011), rendering small inbred populations particularly vulnerable to stressful conditions (Reed et al., 2002; Bijlsma and Loeschcke, 2012). Individuals may be inbred because their parents are close relatives or because they are members of a small population where all individuals share a high degree of coancestry. In either case, increased inbreeding increases the probability that two gene copies chosen at random are identical by descent, measured by the inbreeding coefficient (F) (Wright, 1931). The result is often inbreeding depression (ID), a reduction in fitness primarily due to the expression of recessive deleterious alleles (Charlesworth and Willis, 2009). Relative to benign conditions, environmental stress is predicted to exacerbate ID by increasing the number of alleles with deleterious effects and/or by amplifying the effects of deleterious alleles already expressed under benign conditions (Bijlsma et al., 1999; Reed et al., 2012). However, periods of stress may reverse the buildup of ID through a process of genetic purging. Purging is a reduction in the frequency of deleterious recessive alleles owing to their increased exposure to selection under inbreeding (Hedrick, 1994; Wang, 2000) and has been proposed as a potential tool for managing ID in endangered and captive populations (Swindell and Bouzat, 2006; de Cara et al., 2013). The effectiveness of purging in a population is predicted to increase during exposure to stress, given that stress can magnify the strength of selection against deleterious alleles (Bijlsma et al., 1999; Plough, 2012; Reed et al., 2012).

Although ID is a common phenomenon in nature, understanding what factors contribute to the high degree of variability in magnitude observed across environments remains a challenge (Armbruster and Reed, 2005; Fox and Reed, 2011; Yun and Agrawal, 2014). Predicting the outcome of inbreeding-stress interactions centers on the concept that stress alters the strength of selection against deleterious alleles that cause ID (Agrawal and Whitlock, 2010; Yun and Agrawal, 2014). The finding that the intensity of stress scales positively with ID in a variety of taxa (Fox and Reed, 2011; Enders and Nunney, 2012; Schou et al., 2015) supports the general prediction that selection is greater in

${ }^{1}$ Department of Biology, University of California, Riverside, Riverside, CA, USA and ${ }^{2}$ Department of Entomology, University of Nebraska-Lincoln, Lincoln, NE, USA Correspondence: Dr LS Enders, Department of Entomology, University of Nebraska-Lincoln, 103 Entomology Hall, Lincoln, NE 68583, USA.

E-mail: lenders2@unl.edu

Received 16 June 2015; revised 5 October 2015; accepted 6 October 2015; published online 25 November 2015 
stressful environments. However, different stress types have been shown to vary in their ability to increase selection against mutations (Agrawal and Whitlock, 2010). Recent work in Drosophila also found the degree of density-dependent intraspecific competition associated with a stressful environment was a better predictor of ID than the level of stress alone (Yun and Agrawal, 2014), further suggesting that stressor-specific factors influence the expression of ID.

Environmental stress can be broadly categorized as abiotic or biotic, and within these two groups, there exists a wide range of stress types. Abiotic examples include extreme temperatures, chemicals and desiccation, whereas biotic examples include the presence of predators or parasites, infection with pathogens and intense competition. Organisms often exhibit varied responses to different forms of stress (Kültz, 2005; Atkinson and Urwin, 2012), and plants show characteristic differences in their response to biotic vs abiotic stressors (see Atkinson and Urwin, 2012), suggesting that stressor-specific interactions with the expression of deleterious alleles (that is, the genetic load) may contribute to variation in the expression of ID. Fundamental differences in the way in which unique stressors affect the expression of genes and hence the genetic load could translate into qualitatively different outcomes for the survival of inbred and outbred individuals (Cheptou and Donohue, 2011; Reed et al., 2012). In particular, the potential for biotic stressors to alter the strength and nature of stress imposed on other organisms suggests that their interactions with inbreeding could differ relative to abiotic forms of stress. However, detection of stress-specific effects on ID is difficult unless the level of stress imposed by different stressors is somehow standardized. Ideally, comparison of ID across stress types should be performed using equal stress levels, although regression-based corrections for the variation in the stress level may also be justifiable.

Given the view that stress increases ID through its effects on the expression of deleterious alleles, it is important to know whether these effects cause permanent developmental changes or have a transient effect. The prevailing view is that environmental stress causes general and persistent physiological weakening, the effect of which is greater in inbred individuals. This hypothesis predicts that stress imposed early in life (for example, during development) will lead to increased ID across fitness traits expressed later in life (for example, mating success, fecundity), including periods following direct exposure to stress when conditions are benign (Hoffmann and Parsons, 1991; Armbruster and Reed, 2005; Waller et al., 2008). However, an alternative hypothesis predicts the opposite trend in ID following exposure to stress early in life. This hypothesis invokes intragenerational purging, whereby a period of stress during early life history stages selects against deleterious homozygous genotypes, leading to an increase in the average fitness of the surviving population and subsequent reduction in ID in later life (Enders and Nunney, 2010; Goodrich et al., 2013). Intragenerational stress-induced purging requires that fitness correlations exist across multiple life history stages, such that deleterious mutations affecting early developmental stages also adversely affect adult fitness components, such as mating success or fecundity (Goodrich et al., 2013). These two hypotheses are not exclusive because stress-induced purging and physiological weakening may function simultaneously, with their counteracting effects resulting in little or no change in levels of ID across life history stages or cumulatively over the life span of an organism.

There is some experimental support for the view that short-term exposure to stress at one stage can reduce (or at least not increase) ID at a later developmental stage in plants (Montalvo, 1994; Goodrich et al., 2013) and Drosophila (Enders and Nunney, 2010). In general, it is unknown which (or whether all) types of stress lower ID in later life history stages. Specific stressors can increase, decrease or have no effect on the magnitude of selection against mutations (Agrawal and Whitlock, 2010), and the resulting variation in the effect of different stressors could contribute to differences in the degree of intragenerational purging across stress types (Kristensen et al., 2003; Swindell and Bouzat, 2006). Likewise, differences in the extent to which unique stressors inflict irreversible cellular damage could produce varying degrees of physiological weakening that in turn influence the expression of ID and could counteract the fitness effects of intragenerational purging.

The aim of the present work was to investigate two unresolved aspects of the effect of stress on ID. First, the extent to which different stress types lead to different levels of ID. Second, the extent to which stress imposed early in the life of an organism affects the level of ID expressed later in life, specifically whether ID is increased (the physiological weakening hypothesis) or decreased (the intragenerational purging hypothesis). We investigated these two issues in D. melanogaster by applying four types of environmental stress during the larval stage across three levels of inbreeding $(\mathrm{F}=0,0.25$ and 0.50$)$. We measured ID expressed for larval survival (LS) (reflecting the direct effects of the stress) and for male mating success (post stress). We used male mating success as an indicator of post-stress performance because we had previously shown that the effects of sexual selection exacerbated the expression of ID, so that the effect on males was much greater than that on females (Enders and Nunney, 2010). To compare the effect of the four different kinds of stress, an important feature of the experimental design was that we imposed approximately equal levels of stress with all of the stressors, where the level of stress was defined as the drop in LS observed in the stressed outbred population.

\section{MATERIALS AND METHODS}

\section{Base population and inbreeding design}

In October 2008, outbred stock populations of D. melanogaster were collected from two locations in Northern California, the Galante Winery in Carmel Valley (36 $24^{\prime} 4.827^{\prime \prime} \mathrm{N}, 121^{\circ} 39^{\prime} 26.476^{\prime \prime} \mathrm{W}$ ) (Gala) and the Mayo Family Winery in Sonoma Valley (38 $22^{\prime} 13.868^{\prime \prime} \mathrm{N}, 122^{\circ} 21^{\prime} 0.13^{\prime \prime}$ W) (Mayo). To minimize any modification of the genetic architecture through selection or inbreeding, 400 pairs of wild caught flies from each location were placed in vials and reared in the laboratory at $18^{\circ} \mathrm{C}$. Their progeny were outcrossed by taking a single male and female from each of the 400 pairs (per population) and mating them in a circular design, whereby each male is mated to the female from the next vial. Each of the two outbred stock populations (Gala and Mayo) were reared at $18{ }^{\circ} \mathrm{C}$ using the above circular outcrossing design for six generations prior to the start of the experiment. As each pair contributed equally to the next generation, laboratory selection was minimized because it was restricted to within-family effects, and because the male and female of each pair was unrelated, inbreeding was also minimized.

From both of the large outbred stock populations described above, 10 inbred lines were created for each of two levels of inbreeding $(F=0.25,0.5)$ using the appropriate number of generations of full-sib mating. To create each of these inbred lines ( 2 source populations $\times 2$ levels of $\mathrm{F} \times 10$ replicate lines), five replicate full-sib pairs were set up in separate vials each generation. Ten offspring of one of the five pairs, chosen at random, were used to establish the next generation of sibling mating (five new sibling pairs). This method of sibling mating was carried out for three generations to reach an $\mathrm{F}=0.50$ and for one generation to reach an $\mathrm{F}=0.25$. The level of inbreeding $(\mathrm{F})$ was calculated according to Wright's equation for full-sib mating: $\mathrm{F}_{\mathrm{t}}=\left(1+2 \mathrm{~F}_{\mathrm{t}-1}-\mathrm{F}_{\mathrm{t}-2}\right) / 4$ (Falconer and Mackay, 1996). Both of the two large outbred stock populations $(\mathrm{F}=0)$ were maintained simultaneously as 400 pairs of individuals, using the circular mating design described above. All of the above inbred and outbred lines were reared at $25^{\circ} \mathrm{C}$. 
All inbred lines and the outbred base population were synchronized so that individuals reached the desired level of inbreeding $(\mathrm{F}=0,0.25$ or 0.50$)$ at the same time. When the inbred lines had reached the desired level of $F$, they were expanded to large inbred bottle populations $(N=100)$ to rear enough adults for use in the experiment. This was carried out by taking 100 virgin progeny (50 males: 50 females) from a single sib pair at the final generation of sib mating and placing them into a large bottle to randomly mate. After 1 week, the adults were transferred to a second bottle of new food. The progeny of these two bottles were then used to create two replicate experimental bottle populations $(N=100,50: 50$ virgin males and females) for each of the 20 inbred lines ( 10 each at $\mathrm{F}=0.25$ and 0.50 ) per source population. Five replicate outbred lines (bottle populations with $N=100$ ) were created from each of the two source populations by combining one virgin male and female from each of 50 randomly chosen pairs from the 400 stock pairs used to maintain the outbred populations. The offspring (F1) of the inbred and outbred bottle populations were used in the experimental treatments described below. The experiment was replicated twice (Block I and II) with all lines from both source populations (Gala and Mayo).

\section{Environmental stress treatments}

In all of the 100 tests $(2$ blocks $\times(5$ lines at $\mathrm{F}=0 ; 10$ at $\mathrm{F}=0.25 ; 10$ at $\mathrm{F}=0.5) \times 2$ source populations), the $\mathrm{F} 1$ larvae were subject to four types of stress plus control conditions in vials containing $10 \mathrm{ml}$ of the appropriate food medium. Each condition was replicated three times per test. The control vials contained a standard food medium consisting of molasses, cornmeal, yeast, water and the antifungal agent Tegosept. The abiotic stress treatments were: (i) heat stress, where larvae were reared under fluctuating high temperatures, $28{ }^{\circ} \mathrm{C}$ for $12 \mathrm{~h}$ and $34{ }^{\circ} \mathrm{C}$ for $12 \mathrm{~h}$; and (ii) chemical stress, where larvae were reared on food containing $8.2 \% \mathrm{ETOH}$. The biotic stress treatments were: (i) bacterial stress, where larvae were reared on standard food to which four drops of the pathogenic bacteria Serratia marcescens were added $\left(\sim 4.8 \times 10^{10}\right.$ CFU per vial); and (ii) intraspecific competitive stress, where larvae were reared on food diluted to $33 \%(1 / 3 \times)$ by adding $2 / 3$ agar $\left(18 \mathrm{gl}^{-1}\right)$ to $1 / 3$ of the standard $1 \times$ food medium. The conditions used for each stress treatment were chosen on the basis of preliminary experiments demonstrating an average $45-50 \%$ reduction in the survival of outbred larvae relative to control conditions. Therefore, the level of stress was standardized as much as possible across all four stress treatments.

Another form of standardization was the use of a 'standard' competitor. This provided an internal reference for each vial that can control for a common experimental problem that arises when larval interactions may influence survival. For example, a group of weak competitors can have the same average survival as a group of strong competitors, whereas a mixture of the two would show a substantial survival inequality (Gale, 1964). The use of the standard competitor allows such large differences to be detected.

To set up the experimental vials, each inbred and outbred bottle population described above was transferred to an empty glass bottle capped with a petri dish containing standard food medium and allowed to lay eggs for a period of $8 \mathrm{~h}$. First instar larvae were collected in groups of 100 larvae for up to $8 \mathrm{~h}$ from the laying dish, so that all larvae were within $\pm 4 \mathrm{~h}$ in age. For each experimental vial, larvae were transferred using a paintbrush to vials containing $10 \mathrm{ml}$ of food medium in the ratio of 100 experimental larvae to 150 of a standard competitor spa (D.mel laboratory stock with recessive spa eye mutation). All vials were maintained at $25^{\circ} \mathrm{C}$ except for the heat-stressed vials.

\section{Fitness measures}

Larval to adult survival was measured under the four stress and the benign control conditions. Males that survived these treatments were used to measure mating success.

Larval to adult survival. Following the set up of first instar larva in the experimental vials (day 1), eclosing adults first appeared on days $9-10$ at $25^{\circ} \mathrm{C}$ or on days $8-9$ at $33 / 28{ }^{\circ} \mathrm{C}$. All emerging adults were counted and removed every $3-4$ days until approximately day $20-21$ at $25^{\circ} \mathrm{C}$ and day $19-20$ at $33 / 28^{\circ} \mathrm{C}$, by which time the number of first generation progeny emerging per vial had typically diminished to zero over the final 3-4-day counting interval (day 19-21 or $\sim 18-20$, respectively, for temperature regimes) and a large number of dark pupae representing the next generation were observed. There was always a clear distinction between the first and second generation.

Larval-adult survival was measured in two ways: as the proportion of test larvae surviving to eclosion (LS) and as the larval competitive index (LCI), which is the proportion that eclosed per vial of the test line relative to the proportion of spa competitors (Knight and Roberston, 1957).

Male mating success. Adult males that emerged from the larval-adult survival assay were used to measure male mating success using methods described by Enders and Nunney (2010). In summary, five virgin males were randomly selected from each vial and held in a fresh vial until they were 5-8 days old, when they were placed with 15 unrelated virgin competitor spa males and 10 unrelated virgin spa females in new vials containing $10 \mathrm{ml}$ food for $2 \mathrm{~h}$ at $25^{\circ} \mathrm{C}$. Females were removed using light anesthesia and transferred individually to new vials. After $\sim 2$ weeks, the progeny of each spa female were scored for eye color to determine her mate (100\% wild type if mated to a test male, $100 \%$ spa if mated to spa competitor male).

Male mating success was measured both as a proportion of test males mating (MS) and, relative to the standard competitor spa, as the male competitive index (MCI), which was defined as the proportion of females inseminated by test males divided by the proportion of females inseminated by the standard competitor spa males.

Cumulative male fitness was calculated by multiplying LS (LCI) and male mating success (MCI) for each of the 100 tests described above under each experimental treatment (four stresses and control).

\section{Data analyses}

All analyses below were performed in SAS Version 9.1 for Windows. In all analyses of covariance (ANCOVA), interactions between the continuous variable and all categorical variables were first confirmed to be nonsignificant $(P>0.05)$ before proceeding. In all ANCOVA and analyses of variance (ANOVA), highly nonsignificant interactions $(P>0.25)$ among categorical variables were excluded from the final model.

We calculated the stress level (S_LEVEL) according to Fox and Reed (2011) using the absolute LS of the outbred lines under benign control conditions $\left(\mathrm{LS}_{\text {benign }}\right)$ relative to their survival under each of the four stress treatments $\left(\mathrm{LS}_{\text {stress }}\right)$;

$$
\mathrm{S}_{\mathrm{LEVEL}}=1-\left(\mathrm{LS}_{\text {stress }} / \mathrm{LS}_{\text {benign }}\right)
$$

where, within each experimental block of each source population, $\mathrm{LS}_{\text {stress }}$ was estimated as the mean of the five test values corresponding to the average of three replicates per outbred line for each of the four stress types, and $L S_{\text {benign }}$ likewise for the control conditions, giving 16 total values of S_LEVEL. The experimental design does not allow for meaningful pairing of individual control and stress replicates to calculate stress levels; we therefore averaged across replicates for each outbred line to calculate stress level. By definition, the control treatment had a stress level equal to zero. To determine whether S_LEVEL differed across the stress treatments, we used a one-way ANOVA with STRESS (Heat, Ethanol, Bacteria, Competition), and post hoc multiple comparisons were made using a Tukey test. LCI was not used to estimate stress level because it is a relative measure, that is, if both the outbred and spa larvae responded equally to stress, then the calculated stress level would always be zero.

We also measured post-exposure effects of a stressful larval rearing environment using outbred male mating success $\left(\mathrm{MCI}_{\text {outbred }}\right)$ and cumulative male fitness $\left(\mathrm{LCI} \times \mathrm{MCI}=\mathrm{CUM}_{\text {outbred }}\right)$. S_EFFECT was calculated as the relative reduction in the fitness $\left(\mathrm{MCI}_{\text {outbred }}\right.$ or $\left.\mathrm{CUM}_{\text {outbred }}\right)$ of outbred males reared under stressful and benign conditions:

$$
S_{\text {EFFECT }}=1-\left(\text { Fitness }_{\text {stress }} / \text { Fitness }_{\text {benign }}\right)
$$

where, within each block of each source population, Fitness stress $_{\text {was }}$ estimated for each of the four stress types and Fitness ${ }_{\text {benign }}$ for the control conditions by averaging across the five test values calculated from five outbred lines, giving 16 total values of S_EFFECT. To determine whether S_EFFECT calculated for both $\mathrm{MCI}_{\text {outbred }}$ and $\mathrm{CUM}_{\text {outbred }}$ differed across the stress treatments, we used a 
one-way ANOVA, and post hoc multiple comparisons were made using a Tukey test adjusted for multiple testing.

ID was analyzed for both LS and male mating success using the raw percentage of larvae surviving (LS) or males mating (MS), the competitive index ( $\mathrm{LCI}$ or $\mathrm{MCI}$ ), and the multiplicative measure of cumulative male fitness $(\mathrm{LCI} \times \mathrm{MCI})$. Overall conclusions did not differ between analyses of raw percentages (LS or MS) and competitive indices (LCI and MCI), therefore we only report results using LCI and MCI for analysis of difference in ID. The competitive indices were log-transformed and used to calculate the number of lethal equivalents $(\beta)$, a measure commonly used to compare the effects of inbreeding on fitness across studies, species/taxa and environments (Armbruster and Reed, 2005) using:

$$
-\ln \text { (fitness) }=\mathrm{bF}+c
$$

(Morton et al, 1956), where $\mathrm{F}$ is the level of inbreeding ( $\mathrm{F}=0,0.25$ or 0.50$)$, fitness was either LCI, MCI or cumulative male fitness, and $\mathrm{c}$ is the intercept. We calculated the number of lethal equivalents for both populations across five environmental treatments in two replicate blocks of the experiment (20 total values). This approach assumes that deleterious loci have independent effects on fitness and predicts $\ln$ (fitness) decreases linearly with increased inbreeding. We tested the linearity of regression (3) by comparing the first and second order polynomial regressions using an $\mathrm{F}$ test to determine whether inclusion of a quadratic term significantly improved model fit (that is, there was curvature in the relationship between ID and F level).

Although we attempted to standardize the stress levels imposed (the reduction in outbred LS under stressed relative to control conditions), variation was to be expected. To determine the potential effect of variation in larval stress level on ID, we performed two analyses: (i) ANCOVA on levels of ID $(\beta)$ for LCI, MCI and cumulative fitness across the four stressful larval treatments (STRESS: heat, ethanol, bacteria and competition) with S_LEVEL calculated using equation (1) as a covariate and (ii) ANCOVA on levels of ID ( $\beta$ ) for LCI, MCI and cumulative fitness across the five experimental treatments (ENV: control, heat, ethanol, bacteria and competition) using larval S_LEVEL as a covariate. In this second ANCOVA, $\beta$ and S_LEVEL were calculated for each environment (including the control conditions) within each of the 4 block/population combinations (20 total), and stress level was defined by:

$$
S_{\mathrm{LEVEL}}=1-\left(\mathrm{LS}_{\mathrm{ENV}} / \operatorname{Avg} \mathrm{LS}_{\mathrm{Benign}}\right)
$$

where Avg $\mathrm{LS}_{\text {benign }}$ was the average of all control replicates across the 2 blocks $\times 2$ populations. This differed from the calculations based on equation (1), which defined each stress level relative to its block $\times$ population control $\left(\mathrm{LS}_{\text {benign }}\right)$, thus defining all control S-LEVEL as zero.

To determine whether the number of lethal equivalents differed under benign $\left(\beta_{\text {benign }}\right)$ and stressful $\left(\beta_{\text {stress }}\right)$ conditions, an ANOVA was run for each fitness measure (LCI, MCI, cumulative fitness) using the following variables: ENV (Control, Heat, Ethanol, Bacteria, Competition), POP (Gala,Mayo) and BLOCK. ENV was a fixed effect, whereas BLOCK and POP were random variables. The following a priori independent planned comparisons of lethal equivalents were performed: (i) Control (Benign) vs Stress Conditions; (ii) Abiotic vs Biotic Stresses; (iii) Ethanol vs Heat; and (iv) Bacteria vs Competition. Post hoc multiple comparisons were calculated using a Tukey test with Games and Howell's correction for unequal variances to determine whether ID differed across the environmental treatments.

Intragenerational purging of genetic load predicts a negative correlation between the level of ID ( $\beta$ ) for LS and male mating success (MCI). LS is the relevant variable in this prediction (instead of LCI), because LS is a direct measure of the potential purging. We calculated the correlation between ID for LS and ID for male mating success, using each population (Gala and Mayo), block (Block I and II) and environmental condition as data points. An ANCOVA was also run to determine whether ID post exposure to stress (B_MCI: ID measured as lethal equivalents for male mating success) was affected by ID during the larval period (B_LS: lethal equivalents for larval survival), category of stress (S_CATEGORY: abiotic vs biotic) and the specific STRESS nested within S_CATEGORY (that is, Heat vs Ethanol; Bacteria vs Competition).

We also tested for purging effects in the outbred populations by comparing post-exposure stress effects in males and stress levels during larval exposure. An ANCOVA was run to determine whether stress effects in males (S_EFFECT) were affected by stress levels during the larval period (S_LEVEL), category of stress (S_CATEGORY: abiotic vs biotic) and the specific STRESS nested within S_CATEGORY.

\section{RESULTS}

Stress level: exposure and post-exposure fitness effects on outbred individuals

Stress level was quantified as the decrease in survival of outbred larvae subject to stress relative to their survival under benign conditions (equation (1) using the absolute level of LS). The mean stress level across all four stress types was $0.42 \pm 0.03$, that is, there was an average $42 \%$ reduction in outbred LS relative to benign conditions. ANOVA revealed significant variation in the level of larval stress induced by the four stressors (Figure 1: $\mathrm{F}_{3,12}=5.33, P=0.01$ ). Stress level was homogeneous across three of the four stressors (averaging $0.46 \pm 0.02$ across ethanol, bacteria and larval competition), but significantly lower for the high temperature treatment $(0.29 \pm 0.01)$.

We quantified the post-exposure effects of a stressful larval environment using the relative competitive mating success (MCI) of outbred males reared in benign and stressful conditions (equation (2)). Experiencing stress during larval to adult development reduced male mating success across all four stressors by an average of $57 \pm 3 \%$. This post-exposure reduction in outbred male fitness relative to benign conditions (post-exposure effects of stress) varied significantly across the different stress types (Figure 1: $F_{3,12}=32.6, P<0.001$ ). Post hoc testing indicated the biotic stresses (competition and bacteria) induced equivalent post-exposure loss of fitness $(0.57 \pm 0.02)$. Among the abiotic stressors, heat induced a greater post-exposure reduction in fitness than all other stressors $(0.79 \pm 0.01, P=0.01)$, whereas ethanol caused less of an effect post exposure $(0.35 \pm 0.02, P=0.01-0.02)$.

The overall effect of early developmental (larval) stress was quantified as changes in cumulative outbred male fitness, a measure reflective of both direct and residual effects of larval stress. On average, cumulative outbred male fitness was reduced by $50 \pm 3 \%$ across all stressors relative to benign conditions. However, there was a significant variation in this fitness reduction across stress types $\left(\mathrm{F}_{3,12}=6.06, P<0.01\right)$, owing to males exposed to bacterial stress performing significantly worse than those exposed to ethanol $(0.60 \pm 0.09$ vs $0.43 \pm 0.06, P<0.01$; Figure 1$)$.

\section{ID during exposure to stress}

Stress imposed during the larval period always resulted in greater ID than under benign conditions, measured as decreased LS in inbred lines (Table 1a). Assuming that ID is due to a set of independently acting loci, it can be converted to lethal equivalents using the regression equation (3). To test this assumption, we examined the linearity of the regression (Table 1). There were no indications of nonlinearity when heat, bacteria or competition were the stressors; however, under control conditions and ethanol stress, there was a drop-off in the effect of increasing inbreeding from $\mathrm{F}=0.25$ to 0.5 compared with $\mathrm{F}=0$ to 0.25 ( $P<0.001,0.01$, respectively).

We examined the effects of larval rearing environment on levels of ID by performing an ANOVA on lethal equivalents derived using the LCI $\left(\beta_{\mathrm{LCI}}\right)$, followed by a series of planned comparisons within the ANOVA that partitioned the effect of each type of stress into hypothesis-testing components (Figure 2a; Table 2a). The planned comparison of $\beta_{\mathrm{LCI}}$ in control vs the four stress conditions demonstrated that the overall effect of stress was significant $(P<0.01)$. Under the four stressful conditions, the average number of lethal equivalents expressed for LS was threefold higher $\left(\beta_{\mathrm{LCI}}=1.47 \pm 0.18\right)$ relative to the benign control conditions $\left(\beta_{\mathrm{LCI}}=0.46 \pm 0.01\right)$ 


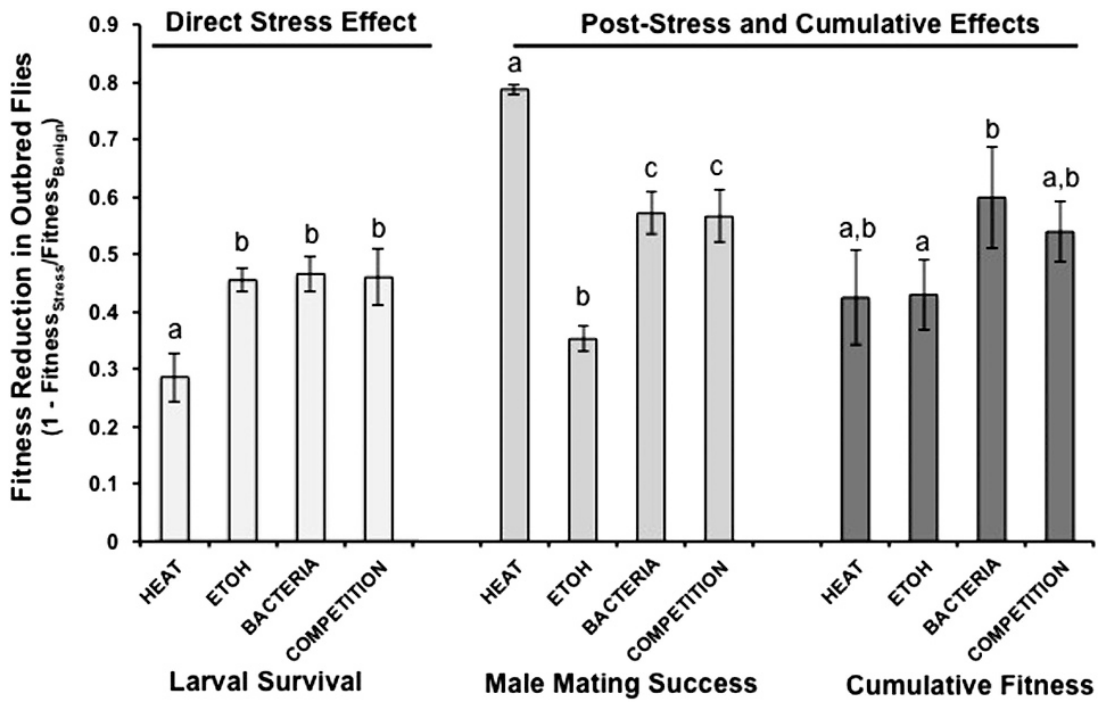

Figure 1 The level of stress measured as a reduction in the fitness of outbred individuals relative to control flies. Direct effects of stress were measured using LS resulting from exposure to each stressor (heat, ethanol, bacteria and larval competition), and the post-exposure effects of stress were measured using the $\mathrm{MCl}$. The two fitness effects were combined $(\mathrm{LCl} \times \mathrm{MCl})$ to estimate the cumulative effect of stress on male fitness. Letters indicate significant differences between stress types for each fitness measure $(P<0.05)$.

Table 1 Summary of the mean ( \pm s.e.) larval survival and male mating success under benign and stressful conditions under three levels of inbreeding

\begin{tabular}{|c|c|c|c|c|c|c|}
\hline Inbreeding & & Control & Heat & $\mathrm{ETOH}$ & Bacteria & Competition \\
\hline \multicolumn{7}{|l|}{ a. Larval survival } \\
\hline \multirow[t]{3}{*}{$\mathrm{F}=0$ (Outbred) } & $\mathrm{LCl}$ & $1.64 \pm(0.03)$ & $3.85 \pm(0.12)$ & $1.44 \pm(0.04)$ & $1.20 \pm(0.02)$ & $1.78 \pm(0.10)$ \\
\hline & \% survival ( LS) & $86.58 \pm(0.67)$ & $62.02 \pm(1.41)$ & $47.46 \pm(1.60)$ & $45.73 \pm(1.32)$ & $47.60 \pm(1.65)$ \\
\hline & & $N=52$ & $N=53$ & $N=54$ & $N=52$ & $N=42$ \\
\hline \multirow[t]{3}{*}{$F=0.25$} & $\mathrm{LCl}$ & $1.34 \pm(0.02)$ & $2.70 \pm(0.08)$ & $1.07 \pm(0.04)$ & $0.90 \pm(0.34)$ & $1.17 \pm(0.07)$ \\
\hline & \% survival (LS) & $75.02 \pm(0.92)$ & $49.79 \pm(1.05)$ & $37.10 \pm(1.63)$ & $34.58 \pm(1.50)$ & $32.17 \pm(1.51)$ \\
\hline & & $N=92$ & $N=94$ & $N=96$ & $N=88$ & $N=59$ \\
\hline \multirow[t]{3}{*}{$F=0.50$} & $\mathrm{LCl}$ & $1.29 \pm(0.02)$ & $1.86 \pm(0.08)$ & $1.00 \pm(0.04)$ & $0.70 \pm(0.04)$ & $0.72 \pm(0.05)$ \\
\hline & \% survival (LS) & $72.70 \pm(0.97)$ & $37.16 \pm(1.38)$ & $36.41 \pm(1.74)$ & $28.41 \pm(1.70)$ & $22.12 \pm(1.44)$ \\
\hline & & $N=85$ & $N=90$ & $N=92$ & $N=81$ & $N=60$ \\
\hline \multirow[t]{2}{*}{$\beta$} & $\mathrm{LCl}$ & $0.46 \pm(0.01)$ & $1.65 \pm(0.32)$ & $0.92 \pm(0.15)$ & $1.19 \pm(0.36)$ & $2.12 \pm(0.18)$ \\
\hline & \% survival (LS) & $0.34 \pm(0.05)$ & $1.14 \pm(0.29)$ & $0.62 \pm(0.22)$ & $1.16 \pm(0.38)$ & $1.80 \pm(0.31)$ \\
\hline Curvature & $\mathrm{F}_{1,9}(P$ value $)$ & $23.19(<0.001)$ & 0.03 (NS) & $22.53(<0.01)$ & 0.67 (NS) & 0.32 (NS) \\
\hline \multicolumn{7}{|c|}{ b. Male mating success } \\
\hline$F=0$ & $\mathrm{MCl}$ & $3.17 \pm(0.25)$ & $0.67 \pm(0.04)$ & $2.04 \pm(0.20)$ & $1.34 \pm(0.13)$ & $1.35 \pm(0.09)$ \\
\hline \multirow[t]{2}{*}{ (Outbred) } & $\%$ Matings & $71.45 \pm(0.02)$ & $37.93 \pm(0.02)$ & $60.1 \pm(0.02)$ & $51.86 \pm(0.02)$ & $54.56 \pm(0.02)$ \\
\hline & & $N=52$ & $N=54$ & $N=53$ & $N=53$ & $N=42$ \\
\hline \multirow[t]{3}{*}{$F=0.25$} & $\mathrm{MCl}$ & $1.21 \pm(0.09)$ & $0.41 \pm(0.03)$ & $1.07 \pm(0.08)$ & $0.93 \pm(0.07)$ & $0.94 \pm(0.06)$ \\
\hline & $\%$ Matings & $50.74 \pm(0.01)$ & $26.79 \pm(0.01)$ & $46.92 \pm(0.02)$ & $43.85 \pm(0.02)$ & $45.60 \pm(0.02)$ \\
\hline & & $N=84$ & $N=86$ & $N=82$ & $N=75$ & $N=59$ \\
\hline \multirow[t]{3}{*}{$F=0.50$} & $\mathrm{MCl}$ & $0.97 \pm(0.08)$ & $0.31 \pm(0.03)$ & $0.88 \pm(0.08)$ & $0.82 \pm(0.10)$ & $0.67 \pm(0.05)$ \\
\hline & $\%$ Matings & $45.09 \pm(0.02)$ & $21.36 \pm(0.01)$ & $42.05 \pm(0.02)$ & $39.94 \pm(0.02)$ & $36.90 \pm(0.02)$ \\
\hline & & $N=80$ & $N=77$ & $N=70$ & $N=57$ & $N=48$ \\
\hline \multirow[t]{2}{*}{$\beta$} & $\mathrm{MCl}$ & $2.32 \pm(0.23)$ & $1.70 \pm(0.28)$ & $1.67 \pm(0.37)$ & $1.11 \pm(0.15)$ & $1.51 \pm(0.14)$ \\
\hline & $\%$ matings (MS) & $4.20 \pm(0.03)$ & $3.55 \pm(0.07)$ & $4.04 \pm(0.05)$ & $3.90 \pm(0.05)$ & $4.00 \pm(0.01)$ \\
\hline Curvature & $\mathrm{F}_{1,9}(P$ value $)$ & $11.39(<0.001)$ & 0.39 (NS) & 3.06 (NS) & 1.43 (NS) & $<0.01$ (NS) \\
\hline
\end{tabular}


a

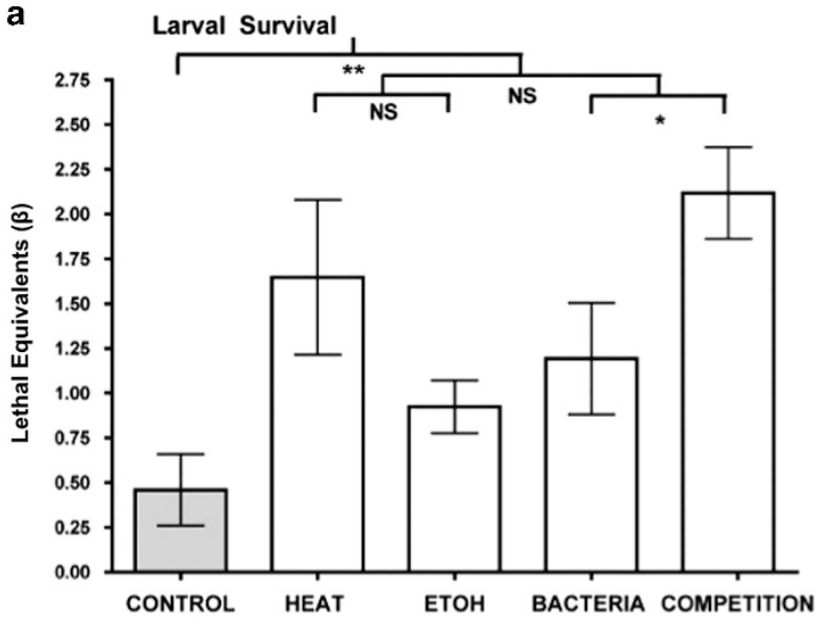

b

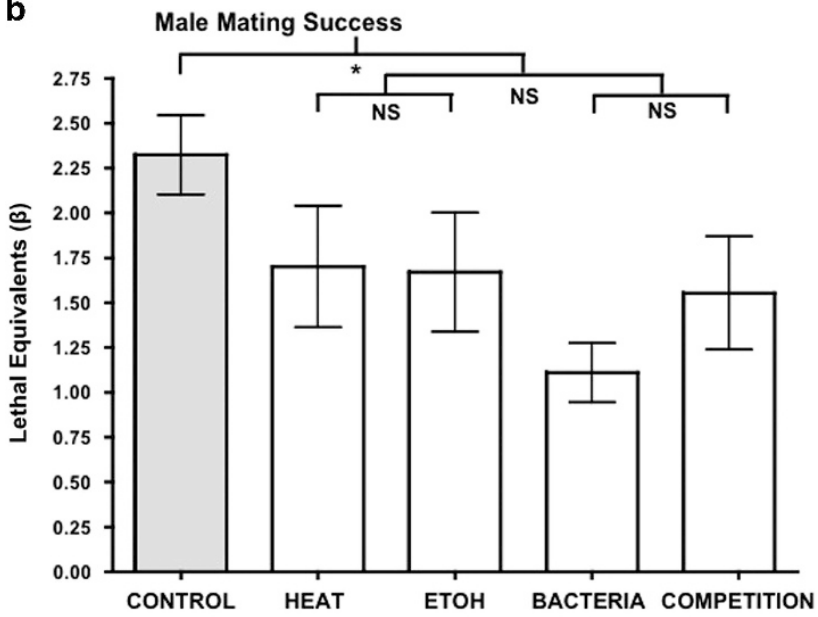

C

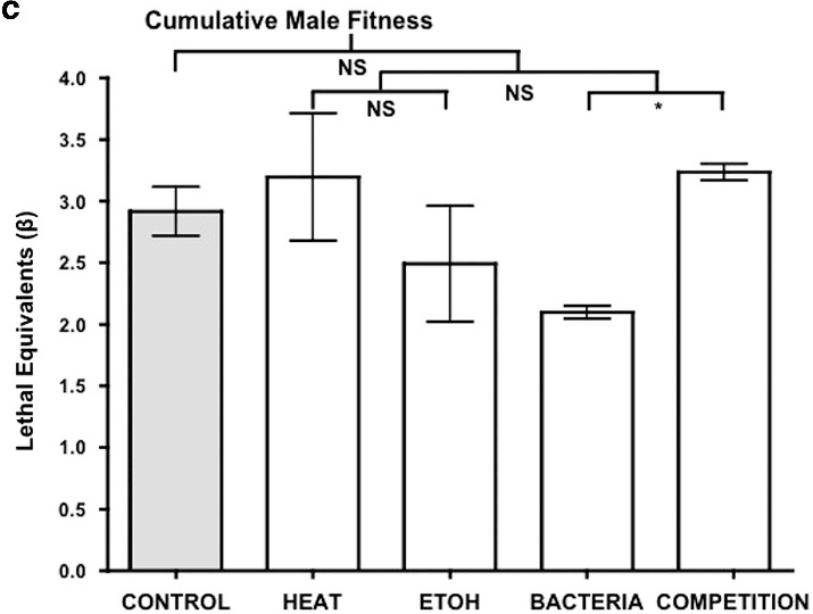

Figure 2 ID for LS and male mating success under benign and stressful conditions. The number of haploid lethal equivalents $(\beta \pm$ s.e.) was measured relative to a standard competitor using (a) the $\mathrm{LCl}$, (b) the $\mathrm{MCl}$ and (c) cumulative male fitness $(\mathrm{LCl} \times \mathrm{MCl})$. The number of lethal equivalents for the control conditions and each individual stress treatment are shown. Significant differences in levels of ID $(\beta)$ were determined using post hoc planned comparisons, which are represented in a tree (NS $P>0.05$, $\left.{ }^{*} P<0.05,{ }^{*} P<0.01,{ }^{* * *} P<0.001\right)$.
Table 2 ANOVA comparing inbreeding depression under benign and stressful conditions for (a) larval survival ( $\mathrm{LCl}$ ), (b) male mating success $(\mathrm{MCl})$ and $(c)$ cumulative male fitness ${ }^{a}$

\begin{tabular}{|c|c|c|c|c|}
\hline Source & $D F$ & $M S$ & $F$ & $\mathrm{P}$ value \\
\hline \multicolumn{5}{|l|}{ a. Larval survival } \\
\hline ENV & 4 & 1.644 & 6.09 & 0.005 \\
\hline Stress vs Control & 1 & & 10.97 & 0.005 \\
\hline Biotic vs Abiotic & 1 & & 1.84 & 0.195 \\
\hline Ethanol vs Heat & 1 & & 3.51 & 0.080 \\
\hline Larval Competition vs Bacteria & 1 & & 5.74 & 0.030 \\
\hline POP & 1 & 0.776 & 2.88 & 0.114 \\
\hline BLOCK & 1 & 0.188 & 0.70 & 0.420 \\
\hline Error & 13 & 0.270 & & \\
\hline \multicolumn{5}{|l|}{ b. Male mating success } \\
\hline ENV & 4 & 0.755 & 5.43 & 0.017 \\
\hline Stress vs Control & 1 & & 7.45 & 0.016 \\
\hline Biotic vs Abiotic & 1 & & 1.74 & 0.207 \\
\hline Ethanol vs Heat & 1 & & 0.01 & 0.940 \\
\hline Larval Competition vs Bacteria & 1 & & 1.38 & 0.258 \\
\hline POP & 1 & 0.003 & 0.02 & 0.902 \\
\hline BLOCK & 1 & 0.573 & 1.72 & 0.222 \\
\hline Error & 13 & 0.139 & & \\
\hline \multicolumn{5}{|l|}{ c. Cumulative male fitness } \\
\hline ENV & 4 & 0.947 & 2.04 & 0.147 \\
\hline Stress vs Control & 1 & & 0.19 & 0.688 \\
\hline Biotic vs Abiotic & 1 & & 0.29 & 0.599 \\
\hline Ethanol vs Heat & 1 & & 2.31 & 0.149 \\
\hline Larval Competition vs Bacteria & 1 & & 6.04 & 0.030 \\
\hline POP & 1 & 0.295 & 0.64 & 0.439 \\
\hline BLOCK & 1 & 0.112 & 0.24 & 0.632 \\
\hline Error & 13 & 0.436 & & \\
\hline
\end{tabular}

Abbreviations: ANOVA, analysis of variance; DF, degrees of freedom; $\mathrm{LCl}$, larval competitive index; $\mathrm{MCl}$, male competitive index; MS, male mating success. Two populations of D. melanogaster (POP: Gala and Mayo) were used and the experiment was replicated twice (BLOCK). Interactions were nonsignificant $(P>0.25)$ and removed from the models. Bold values indicate $P<0.05$

aThe analysis compares inbreeding depression (lethal equivalents) under control conditions and the four stress treatments (ENV), and includes four independent planned comparisons.

(Table 1a). Three additional planned comparisons were used to determine whether the stress type affected the magnitude of ID (Figure 2a; Table 2a). Overall, abiotic and biotic stresses caused similar levels of ID. Within the biotic stress category, the two stresses differed $(P<0.05)$, with larval competition resulting in higher ID. Although the two abiotic stresses, ethanol and heat, were not significantly different $(P=0.08)$, it was notable that heat did result in a higher level of stress-induced ID even though the level of the heat stress was significantly lower (see above).

Although we attempted to minimize variation in stress levels imposed by the different stressors, it was important to determine whether this variation significantly affected the ID detected. For this purpose, we performed an ANCOVA comparing $\beta_{\mathrm{LCI}}$ across all larval stressors (STRESS: heat, ethanol, bacteria, competition) with larval stress level (S_LEVEL) as a covariate. Initial testing showed the effect of S_LEVEL on ID did not vary across stressors (STRESS $\times$ S_LEVEL, $\left.\mathrm{F}_{1,8}=2.15, P=0.17\right)$ and we excluded the interaction. The simplified model showed no significant effect of stress levels (S_LEVEL, $\mathrm{F}_{1,11}=2.65, P=0.10$ ), and ID did not vary significantly across these larval environments, (STRESS, $\mathrm{F}_{3,11}=2.65, P=0.10$ ). Adding the control data to the analysis showed a significant effect of S_LEVEL 
$\left(\mathrm{F}_{1,14}=6.68, P=0.02\right)$, an effect driven by the inevitable near zero values in the benign controls. With inclusion of control data, ID varied significantly across larval environments $\left(\mathrm{F}_{4,14}=3.48, \quad P=0.04\right)$, in agreement with the original ANOVA of $\beta_{\mathrm{LCI}}$ showing stress increased ID relative to control conditions.

\section{ID post exposure to stress}

The effect of stress imposed during the larval period always reduced the ID seen in subsequent male mating success, as measured by MCI or MS (Table 1b). Tests of the regression equation (3) linking ID to lethal equivalents revealed linearity of the fit to equation (3) in the groups previously subjected to stressful conditions (Table 1); however, the controls showed a drop-off in the accumulation of ID at the higher level of inbreeding $(P<0.001)$. Planned comparisons within the ANOVA analysis of ID for male mating $\left(\beta_{\mathrm{MCI}}\right)$ showed that this post-stress reduction was significant $(P<0.02)$ and there were no additional effects due to differences among the stressors (Table 2b; Figure 2b). The number of lethal equivalents for competitive mating success expressed in males exposed to stressful conditions $\left(\beta_{\mathrm{MCI}}=1.60 \pm 0.14\right)$ was on average $30 \%$ lower than males reared under benign conditions $\left(\beta_{\mathrm{MCI}}=2.32 \pm 0.23\right.$ ) (Table $\left.1 \mathrm{~b}\right)$. Exposure to abiotic and biotic stress caused similar levels of ID for male mating success, as did the two abiotic stressors (heat and ethanol) and two biotic stressors (bacteria and competition) (Table 2b).

We further analyzed $\beta_{\mathrm{MCI}}$ using an ANCOVA including the larval stressors (STRESS: heat, ethanol, bacteria, competition) and S_LEVEL experienced during the larval exposure to these stressors to determine whether the experimental variation in stress levels experienced as a larva resulted in variation in the magnitude of ID expressed as an adult male. Initial testing showed no interaction between these two variables (STRESS $\times$ S_LEVEL, $F_{3,8}=0.01$, $P>0.25)$ and the simplified model showed that the level of ID for male mating $\left(\beta_{\mathrm{MCI}}\right)$ varied with larval S_LEVEL $\left(\mathrm{F}_{1,11}=5.12\right.$, $P=0.04$, slope $=-4.12 \pm 1.72$ ), but not the specific stressor (STRESS: $\mathrm{F}_{3,11}=1.35, P=0.31$ ). Adding the control data increased the significance of $\mathrm{S} \_$LEVEL $\left(\mathrm{F}_{1,14}=15.10, P=0.001\right)$, but again there was no effect of environment (ENV: $F_{1,14}=1.15, P=0.37$ ), where ENV adds the control to the four STRESS environments.

\section{Cumulative ID and intragenerational purging}

After combining the inbreeding effects apparent at the stressed larval stage with those affecting later male mating success, ANOVA showed that exposure to stressful conditions during larval development did not cause any cumulative stress-induced ID of males (Stress vs Control, Table 2c; Figure 2c). The number of cumulative lethal equivalents $\left(\mathrm{B}_{\mathrm{CUM}}\right)$ expressed in males that experienced benign conditions was $2.92 \pm 0.19$ vs $2.76 \pm 0.20$ on average across the four stresses. The additional planned comparisons showed that there was no difference in the level of ID resulting from abiotic vs biotic stresses. However, although there was no significant difference between ID caused by the two abiotic stresses, the two biotic stresses did differ, with bacterial infection resulting in significantly lower ID than larval competition (Table 2c; Figure 2c).

An ANCOVA of cumulative male ID that included larval stress level (S_LEVEL) showed no interaction between S_LEVEL and ENV four stresses and control) $\left(\mathrm{F}_{4,10}=1.37, P=0.31\right)$, and the simplified model showed no effect of S_LEVEL $\left(\mathrm{F}_{1,14}=2.88, P=0.11\right.$, slope $\left.=-4.50 \pm 2.20\right)$ or the five experimental treatments (ENV: $\mathrm{F}_{4,14}=3.00, P=0.06$ ).

We exploited the experimental variance among the replicates across the two source populations and two experimental blocks of each of the four stress treatments to test for evidence of intragenerational purging of genetic load. The prediction is that greater levels of ID during the stressed period $\left(\beta_{\mathrm{LS}}\right)$ should result in lower levels of ID post exposure for male mating success $\left(\beta_{\mathrm{MCI}}\right)$. We used LS rather than LCI (a measure including competitive success) because LS is a direct estimate of potential purging. ANCOVA of lethal equivalents for male mating success showed a significant interaction between S_CATEGORY (abiotic vs biotic) and $\beta_{\mathrm{LS}}\left(\mathrm{F}_{1,8}=9.4, P=0.015\right)$. Thus, the analysis was split by stress category and we found that ID for male mating $\left(\beta_{\mathrm{MCI}}\right)$ was significantly negatively correlated with ID for LS $\left(\beta_{\mathrm{LS}}\right)$ in the biotic stresses $(P=0.04)$, whereas for the abiotic stresses, there was a nonsignificant positive slope (Table 3; Figure 3). Although the two biotic stresses showed the same slope, their elevations differed $(P=0.02)$ possibly indicating more effective purging by the bacterial treatment.

Outbred populations did not show evidence of intragenerational purging, that is, there was no significant relationship between stress level of the outbred larvae and stress effect for mating success of outbred males (Figure 4). ANCOVA of stress effect in male mating (S_EFFECT) showed no indication of a correlation with stress levels experienced as larva (S_LEVEL) (slope $=-0.52 \pm 0.36, \mathrm{~F}_{1,8}=0.48$, $P=0.51)$ and this relationship did not vary across the two broad stress categories (S_LEVEL $\times$ S_CATEGORY: $\mathrm{F}_{1,8}=1.93, P=0.2$ ). Overall, there were no differences between stress categories (S_CATEGORY: $\mathrm{F}_{1,8}=2.15, P=0.18$ ) or across individual stressors $\left(\mathrm{S} \_\right.$TYPE $\left(\mathrm{S} \_\right.$CATEGORY): $\mathrm{F}_{1,8}=1.39, P=0.30$ ).

\section{DISCUSSION}

The questions addressed in this study were: (i) Does stress consistently increase ID in the life history stage subject to exposure? (ii) Does any increase in ID persist beyond the period of stress? (iii) How do these stress effects combine to affect overall fitness in inbred populations? and (iv) Do different stressors affect ID in different ways? Our results demonstrate that exposure to heat, ethanol, pathogenic bacteria and larval competition causes consistent and significant increases in the expression of lethal equivalents for larval-adult survival relative to benign conditions (Figure 2a). However, this increase in ID observed during larval exposure to stress did not extend to later life history stages. ID was significantly reduced for males surviving stressful

Table 3 ANCOVA comparing the level of inbreeding depression (lethal equivalents) for male mating success ( $\mathrm{MCl}$ ) after exposure as larvae to abiotic or biotic stress

\begin{tabular}{lcccc}
\hline Source & DF & MS & $F$ & P value \\
\hline Abiotic stresses & & & & \\
$\quad$ STRESS TYPE & 1 & 0.315 & 1.130 & 0.348 \\
$\quad$ ID_LS & 1 & 1.081 & 3.880 & 0.120 \\
$\quad$ Slope $=0.50 \pm 0.40$ & & & & \\
$\quad$ Error & 4 & 0.278 & & \\
$\quad$ Biotic stresses & & & & \\
$\quad$ STRESS TYPE & 1 & 0.879 & 10.400 & 0.023 \\
$\quad$ ID_LS & 1 & 0.665 & 7.870 & 0.038 \\
$\quad$ Slope $=-0.71 \pm 0.17$ & & & & \\
$\quad$ Error & 4 & 0.278 & & \\
\hline
\end{tabular}

Abbreviations: ANCOVA, analysis of covariance; DF, degrees of freedom; LS, larval survival; $\mathrm{MCl}$, male competitive index; MS, male mating success. The analysis included STRESS TYPE: abiotic stress (heat and ethanol) and biotic stress (bacteria and larval competition), and the level of stress (heat and ethanol) and biotic stress (bacteria and larval competition), and the level
inbreeding depression expressed in larval survival during exposure to stress (ID_LS) as a covariate. The analysis was split by category of stress (abiotic vs biotic) owing to a significant interaction between stress category and ID_LS $\left(F_{1,8}=9.4 P=0.015\right)$. Nonsignificant interactions with the covariate (ID_LS $\times$ STRESS TYPE) were removed and the model was rerun. Bold values indicate $P<0.05$. 


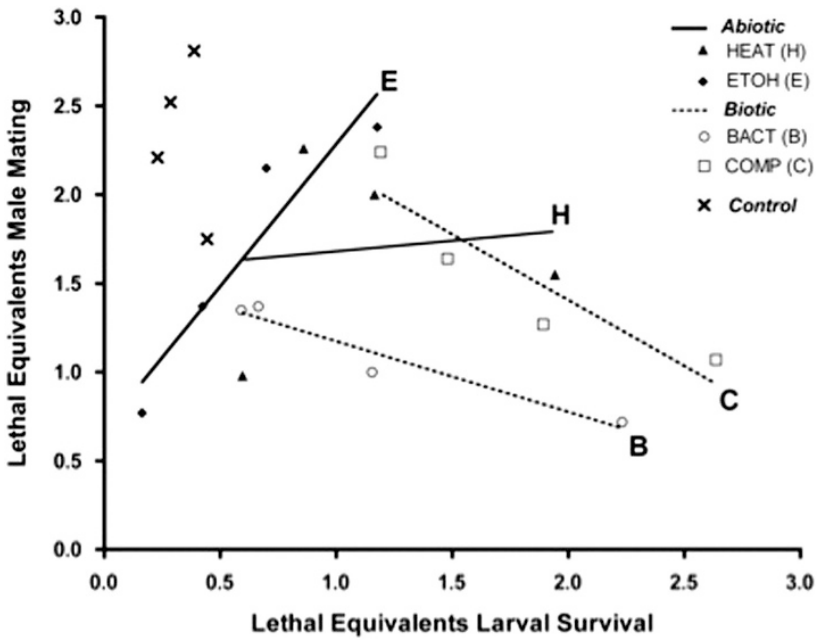

Figure 3 Relationship between ID in lethal equivalents expressed under stress and post stress. ID under stress was estimated using LS $\left(\beta_{\mathrm{LS}}\right)$ and post stress using male mating success $\left(\beta_{\mathrm{MCl}}\right)$. For each of the stress treatments, four points are shown corresponding to the average lethal equivalents for each replicate (Block I and II) in each population (Gala and Mayo). The fitted line is indicated for each stress, although the slopes only differ between biotic and abiotic treatments (Table 3 ).

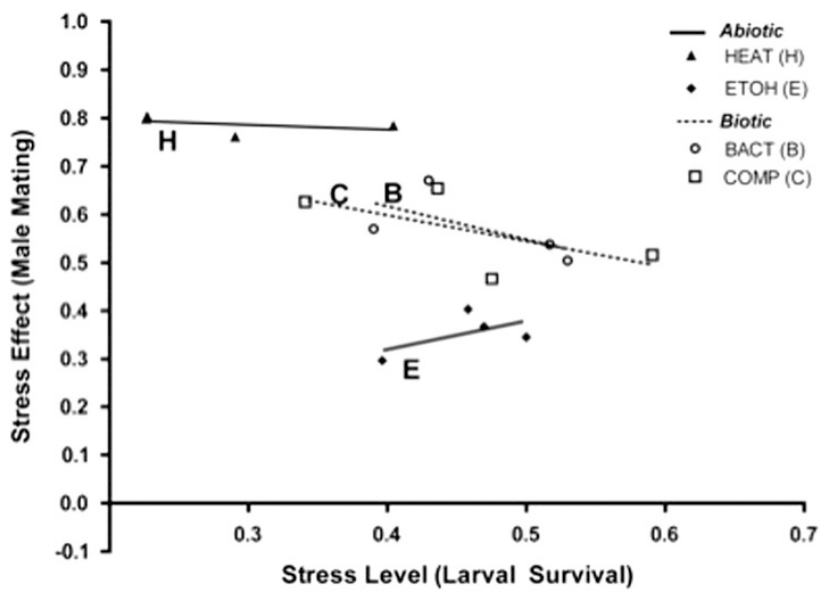

Figure 4 Relationship in outbred individuals between stress level during larval exposure and the post-stress effect after exposure on male mating. Stress level was measured using LS and post-exposure stress effects using male mating success $(\mathrm{MCl})$.

larval conditions compared with those that developed in a benign environment (Figure $2 \mathrm{~b}$ ), indicative of purging of deleterious alleles during exposure to stress. When examined cumulatively in males, we found the paradoxical result that in spite of the large direct effect of stress in increasing larval ID, the net ID was no different under stressful and benign conditions (Figure 2c), suggesting that because of intragenerational stress-induced purging, stress had minimal population-level effects on the overall expression of genetic load.

\section{Is stress type an important determinant of ID?}

The central role of stress level in determining ID raises the question of the extent to which the type of stress matters. Such comparisons of different stressors require that stress level be standardized (Fox and Reed, 2011) or, if not, differences should be accounted for in the analysis. We followed this protocol (with an average stress level of $42 \%$ ) and found no differences in ID when larvae were directly exposed to stress based on the biotic vs abiotic grouping (Table 2), although within these two groups, there were marginally significant differences in the level of ID (competition > bacteria; heat > ethanol; see Figure 2a). After the stress had been removed, previously stressed male flies had significantly lower ID compared with unstressed control flies, an effect that also did not vary by stress type (Tables $1 \mathrm{~b}$ and $2 \mathrm{~b}$; Figure $2 \mathrm{~b}$ ). These results are in line with previous work in Drosophila using a similar level of larval competitive stress (stress level $=52 \%$ ), which found higher ID during exposure (larval-adult survival) but not for post-stress adult reproductive fitness traits (Enders and Nunney, 2010).

We also examined whether different stressors resulted in an accelerating or decelerating expression of ID as inbreeding increased from $\mathrm{F}=0.25$ to $\mathrm{F}=0.5$ relative to expectation (see equation (3)). The ID resulting from the stressors fitted closely to expectation, consistent with deleterious alleles acting independently to reduce fitness. The only exception was the effect of ethanol stress on larval ID. In contrast, we found a highly significant decelerating effect under control conditions both for larval and adult male ID. Thus, there is no evidence that deleterious alleles are acting synergistically under stressful conditions to decrease fitness (that is, there is no negative epistasis), and that under non-stressful control conditions, there is positive epistasis, so that combinations of deleterious alleles are less damaging than would be expected.

Overall, stress type was found to cause only minor differences in both the increase in ID expressed during direct exposure and the decrease in ID observed in post-exposure male mating success (Figures 2a and b). Thus, our results do not support the findings of Yun and Agrawal (2014), who found that ID was strongest when competition was intense. We found that, although competition resulted in a slightly higher ID than the other stressors, its effect was very similar to that of heat, while the other two stressors (ethanol and bacteria) induced very similar levels of ID (see Figure 2a), pairings that suggest no particular pattern. The distinction between biotic and abiotic stressors has been found to be important in the study of plant responses to stress, where different signaling pathways can be involved (see review by Atkinson and Urwin 2012). This is notably the case when biotic and abiotic stressors interact; however, in our experiments, we have not investigated the possibility of such interactions. Although we found no differences in the magnitude of the effect of these two types of stressors, there were indications in the data that abiotic stressors may cause additional damage (see below).

Similarity in the physiological and cellular damage induced by different stressors, as well as overlap in molecular level defenses, could contribute to minimizing differences in the expression of ID across stress types. Although different stressors can have variable effects, similar changes in gene expression and protein abundance have also been observed under a variety of stressors in plants and animals (Kültz, 2005; Storz and Hengge, 2011). For example, work in Drosophila has shown that common molecular responses are present in populations selected for tolerance to a variety of abiotic stressors such as heat, starvation and desiccation (Sørensen et al., 2007). In addition, inbreeding alters the expression of genes involved in primary metabolic processes and general stress defense (Kristensen et al., 2005; Kristensen et al., 2006), which could render individuals generally more vulnerable to a variety of stressors. If molecular mechanisms underlying defense responses were predominately shared among the stress types used in the current study, this would also minimize variation in the expression of ID.

Despite our efforts to equalize the level of stress during larval development across all four types of stress, heat treatment was found 
to be less stressful than the other three stresses (Figure 1). When this variation was accounted for using ANCOVA, the relationship between stress level and ID was not significant for LS or cumulative fitness, indicating that lower mortality under heat stress was not affecting the observed patterns in ID.

Stress-induced intragenerational purging vs physiological weakening Stress is commonly viewed as a cumulative phenomenon, whereby exposure early in life causes an overall physiological weakening that is predicted to amplify ID across multiple life history stages even after the source of stress is removed (Hoffmann and Parsons, 1991; Kristensen et al., 2003; Armbruster and Reed, 2005; Waller et al., 2008). Contrary to this view, our results demonstrated that early exposure to stress consistently reduced ID in later life history stages (Figure 2b). Intragenerational purging appears to be the explanation: exposure to larval stress purges less fit larvae that in turn causes a reduction in genetic load in surviving males, resulting in enhanced performance relative to unstressed individuals.

Intragenerational purging depends on genetic variation within an inbred population and is predicted to occur if early and late fitness components are correlated owing to some genes having pleiotropic effects important for both (Whitlock and Bourguet, 2000; Whitlock and Agrawal, 2009). Maintaining metabolic efficiency under stressful conditions has been hypothesized to be important for energetically costly processes such growth, development and mating success involving courtship and competitive interactions, which predicts stress-induced associations between diverse fitness components (Parsons, 1997; Parsons, 2007). For example, the insulin signaling pathway regulates growth and development in both larva and adult Drosophila (see review in Garofalo, 2002). Thus, recessive deleterious mutations in these pathways could reduce survival and body size, and could therefore adversely affect both LS and male mating success.

Such correlations between fitness components are necessary for intragenerational purging, and predict a negative relationship between ID during the larval period (which measures the opportunity for purging) and ID for male mating success (which measures the consequences of purging). We first eliminated the possibility that such a correlation could arise from a direct relationship between the traits unrelated to purging by showing that in outbred flies, there was no significant negative correlation between larval stress and post-stress mating success relative to the unstressed control (Figure 4). Next, we established that, although abiotic and biotic stressors induced equivalent levels of enhanced larval ID and reduced male mating ID, there were significant differences in the predicted correlation (Table 3; Figure 3). Biotic stressors showed a significant negative relationship consistent with intragenerational purging. Abiotic stressors showed a nonsignificant positive relationship between ID during and after exposure (Figure 3), suggesting that, although purging appeared to be important (causing the reduced ID for male mating success; see Figure 2b), something else was also affecting the outcome. We propose that this pattern seen with the two abiotic stresses could arise from a combination of purging and a general stress-induced physiological weakening. Extensive work in a variety of animals shows longterm protein damage and impaired cellular functioning can result from heat and ethanol stress (Hoffmann and Parsons, 1991; Sorensen et al., 2003; Montooth et al., 2006). Studies in Drosophila found exposure to high heat and sublethal concentrations of ethanol alters membrane lipid composition and energy reserves (Sorensen et al., 2003; Montooth et al., 2006), which could have enduring negative effects on later life history traits such as female fecundity and male fertility (Krebs and Loeschcke, 1994; Bokor and Pecsenye, 2000).
Both heat and ethanol stress also induce upregulation of heat shock proteins in Drosophila (Sorensen et al., 2005; Kong et al., 2010), however, this increased expression can also be costly for growth, development and longevity (see reviews in Sorensen et al., 2003). Therefore, irreversible damage to basic cellular functioning and long-term costs associated with mounting molecular stress defenses could offset in part the fitness benefits associated with stress-induced purging of genetic load under abiotic heat and ethanol stress.

Overall, our demonstration that early-life exposure to different stressors caused increased post-stress fitness is unique, as is our finding of a potentially important difference between biotic and abiotic stressors, with abiotic stressors perhaps inducing a persistent physiological weakening at the stress levels that we examined. It will be interesting to see whether this biotic/abiotic distinction persists in future studies across a range of stress levels. If the stress-induced loss of fitness accelerates relative to the fitness gain via purging as the level of stress increases, then purging is predicted to dominate at low stress levels, but at higher levels, any further benefits of purging would be negated by the increasing stress-induced loss of fitness via irreversible cellular damage. In addition, the purging effect is predicted to decline with increased inbreeding (because purging relies on the presence of genetic variation) but the weakening effect works equally well in a genetically uniform population.

\section{Cumulative patterns of ID}

Combining LS and mating success, it was found that cumulative ID expressed in males was independent of the presence or absence of stress (Table 2c; Figure 2c). The only indication of any effect of stress type on this result was a significant difference between the two biotic stresses, driven mainly by the low cumulative inbreeding in the bacterial treatment. A similar equalization of the cumulative ID was apparent in the study of Montalvo (1994), where early development of perennial Aquilegia caerulea occurred either in the greenhouse or field. However, unlike previous studies where plants were maintained in stressful field conditions following early development (Montalvo, 1994; Goodrich et al., 2013), the most plausible explanation for patterns of cumulative ID observed in the current study is intragenerational purging. We maintained males in a benign environment following developmental stress, thus avoiding the possibility that continued exposure to stressful conditions in later life stages and additional selective pressure could explain the equivalent cumulative ID observed across environments.

Given a correlation of deleterious effects acting during stressful and post-stress life history stages, we can expect some degree of intragenerational purging. However, the magnitude of the effect is hard to predict, because it depends upon the sensitivity of the different fitness components to the presence/absence of deleterious alleles under the conditions being tested. Thus, although it is clear that stress increases such sensitivity (resulting in increased ID), the sexual selection acting on male mating success may, depending upon conditions, result in even greater fitness discrimination. This would lead to the paradoxical result of lower cumulative ID in stressed populations. In our experiments, it appears that the stress-related fitness effects and subsequent mating effects were approximately balanced, leading to the observed result of equal ID in control and stressed groups; although it is interesting to note that the cumulative ID observed following stress with a bacterial pathogen was substantially lower than that observed in the control group (Figure 1c).

Our finding of no net increase in ID with stress when fitness is evaluated cumulatively brings into question the generality of the suggestion that increased stress can increase the efficiency of purging 
deleterious alleles at the population level. This has been proposed as a mechanism useful for alleviating ID and increasing the long-term fitness of threatened species (Leberg and Firmin, 2008; de Cara et al., 2013). In particular, we found despite a substantial direct effect of stress, increasing ID from 0.46 to 1.47 lethal equivalents, the mating success of males surviving larval stress completely eliminated this difference. Under such circumstances, stress-induced purging is unlikely to have beneficial population-level effects.

\section{DATA ARCHIVING}

Data available from the Dryad Digital Repository: http://dx.doi.org/ 10.5061/dryad.t8344.

\section{CONFLICT OF INTEREST}

The authors declare no conflict of interest.

\section{ACKNOWLEDGEMENTS}

We would like to thank Magdalene Moy for help with Drosophila rearing and conducting this experiment. This research was supported in part by an NSF DDIG awarded to L Enders (award no. 0808416).

Agrawal AF, Whitlock M (2010). Environmental duress and epistasis: how does stress affect the strength of selection on new mutaitons? Trends Ecol Evol 25: 450-458.

Armbruster P, Reed DH (2005). Inbreeding depression in benign and stressful environments. Heredity 95: 235-242.

Atkinson NJ, Urwin PE (2012). The interaction of plant biotic and abiotic stresses: from genes to the field. J Exp Bot 63: 3523-3543.

Bijlsma R, Bundgaard J, Van Putten WF (1999). Environmental dependence of inbreeding depression and purging in Drosophila melanogaster. J Evol Biol 12: 1125-1137.

Bijlsma R, Loeschcke V (2012). Genetic erosion impedes adaptive responses to stressful environments. Evol App/ 5: 117-129.

Bokor K, Pecsenye K (2000). Differences in the effect of ethanol on fertility and viability components among laboratory strains of Drosophila melanogaster. Hereditas 132: 215-227.

Charlesworth D, Willis JH (2009). The genetics of inbreeding depression. Nat Rev Genet 10: 783-796.

Cheptou PO, Donohue K (2011). Environment-dependent inbreeding depression: its ecological and evolutionary significance. New Phytol 189: 395-407.

de Cara MÁ, Villanueva B, Toro MÁ, Fernández J (2013). Purging deleterious mutations in conservation programmes: combining optimal contributions with inbred matings. Heredity 110: 530-537.

Enders L, Nunney L (2010). The sex-specific effects if inbreeding in wild-caught Drosophila melanogaster. J Evol Bio 23: 2309-2323.

Enders LS, Nunney L (2012). Seasonal stress drives predictable changes in inbreeding depression in field-tested captive populations of Drosophila melanogaster. Proc Biol Sci 279: 3756-3764.

Falconer DS, Mackay TFC (1996). Introduction to Quantitative Genetics, 4th edn. Longman: New York.

Fox C, Reed DH (2011). Inbreeding depression increases with environmental stress: and experimental study and meta-analysis. Evolution 65: 246-258.

Frankham R (2005). Stress and adaptation in conservation genetics. J Evol Biol 18 $750-755$.

Gale J (1964). Competition between three lines of Drosophila melanogaster. Heredity 19 681.

Garofalo RS (2002). Genetic analysis of insulin signaling in Drosophila. Trends Endocrinol Metab 13: 156-162.

Goodrich S, Beans C, Roach D (2013). Environmental conditions during early life determine the consequences of inbreeding in Agrostemma githago (Caryophyllaceae). J Evol Biol 26: 499-508.

Hedrick PW (1994). Purging inbreeding depression and the probability of extinction: Full-sib mating. Heredity 73: 363-372.
Hoffmann AA, Parsons PA (1991). Evolutionary Genetics and Environmental Stress. Oxford University Press: New York.

Knight GR, Roberston A (1957). Fitness as a measureable character in Drosophila Genetics 42: 524-530.

Kong EC, Allouche L, Chapot PA, Vranizan K, Moore M, Heberlein U et al. (2010). Ethanolregulated genes that contribute to ethanol sensitivity and rapid tolerance in Drosophila. Alcohol Clin Exp Res 34: 302-316.

Krebs RA, Loeschcke V (1994) Response to environmental change: Genetic variation and fitness in Drosophila buzzatii following temperature stress. In: Loeschcke V, Tomiuk J, Jain SK (eds). Conservation Genetics. Basel: Birkhäuser. pp 309-322.

Kristensen TN, Dahlgaard J, Loeschcke V (2003). Effects of inbreeding and environmental stress on fitness - using Drosophila buzzatii as a model organism. Cons Gen 4 453-465.

Kristensen TN, Sorensen P, Kruhoffer M, Pedersen KS, Loeschcke V (2005). Genome-wide analysis on inbreeding effects on gene expression in Drosophila melanogaster. Genetics 171: 157-167.

Kristensen TN, Sorensen P, Pedersen KS, Kruhoffer M, Loeschcke V (2006). Inbreeding by environmental interactions affect gene expression in Drosophila melanogaster. Genetics 173: $1329-1336$.

Kültz D (2005). Molecular and evolutionary basis of the cellular stress response. Annu Rev Physiol 67: 225-257.

Leberg P, Firmin B (2008). Role of inbreeding depression and purging in captive breeding and restoration programmes. $\mathrm{Mol}$ Ecol 17: 334-343.

Loeschcke V, Sorensen JG, Kristensen TN (2004). Ecologically relevant stress resistance: from microarrays and quantitative trait loci to candidate genes-a research plan and preliminary results using Drosophila as a model organism and climatic and genetic stress as model stresses. J Biosci 29: 503-511.

Montalvo A (1994). Inbreeding depression and maternal effects in Aquilegia caerulea, a partially selfing plant. Evolution 75: 2395-2409.

Montooth KL, Siebenthall KT, Clark AG (2006). Membrane lipid physiology and toxin catabolism underlie ethanol and acetic acid tolerance in Drosophila melanogaster. J Exp Biol 209: 3837-3850.

Morton NE, Crow JF, Muller HJ (1956). An estimation of the mutational damage in man from data on consanguineous marriages. Proc Natl Acad Sci USA 42: 855-863.

Parsons PA (1997). Success in mating: a coordinated approach to fitness through genotypes incorporating genes for stress resistance and heterozygous advantage under stress. Behavioral Genetics 27: 75-81.

Parsons PA (2007). Energetic efficiency under stress underlies positive genetic correlations between longevity and other fitness traits in natural populations. Biogerontology 8 : 55-61.

Plough LV (2012). Environmental stress increases selection against and dominance of deleterious mutations in inbred families of the Pacific oyster Crassostrea gigas. Mol Ecol 21: 3974-3987.

Reed DH, Briscoe DA, Frankham R (2002). Inbreeding and extinction: The effect of environmental stress and lineage. Cons Gen 3: 301-307.

Reed DH, Fox CW, Enders LS, Kristensen TN (2012). Inbreeding-stress interactions: evolutionary and conservation consequences. Ann NY Acad Sci 1256: 33-48.

Schou M, Loeschcke V, Kristensen T (2015). Inbreeding depression across a nutritional stress continuum. Heredity 115: 56-62.

Sorensen JG, Kristensen TN, Loeschcke V (2003). The evolutionary and ecological role of heat shock proteins. Ecol Lett 6: 1025-1037.

Sørensen JG, Nielsen M, Loeschcke V (2007). Gene expression profile analysis of Drosophila melanogaster selected for resistance to environmental stressors. J Evol Biol 20: 1624-1636.

Sorensen JG, Nielsen MM, Kruhoffer M, Justesen J, Loeschcke V (2005). Full genome gene expression analysis of the heat stress response in Drosophila melanogaster. Cell Stress Chaperones 10: 312-328.

Storz G, Hengge R (2011). Bacterial Stress Responses. ASM press: Washington, DC, USA.

Swindell WR, Bouzat JL (2006). Ancestral inbreeding reduces the magnitude of inbreeding depression in Drosophila melanogaster. Evolution 60: 762-767.

Waller DM, Dole J, Bersch AJ, Schoen D (2008). Effects of stress and phenotypic variation on inbreeding depression in Brassica rapa. Evolution 62: 917-931.

Wang J (2000). Effects of population structures and selection strategies on the purging of inbreeding depression due to deleterious mutations. Gen Res 76: 75-86.

Whitlock MC, Agrawal AF (2009). Purging the genome with sexual selection:reducing mutation load through selection on males. Evolution 63: 569-582.

Whitlock MC, Bourguet D (2000). Factors affecting the genetic load in Drosophila: Synergistic epistasis and correlations among fitness components. Evolution 54: 1654-1660.

Wright S (1931). Evolution in Mendelian Populations. Genetics 16: 97-159.

Yun L, Agrawal AF (2014). Variation in the strength of inbreeding depression across environments: Effects of stress and density dependence. Evolution 68: 3599-3606. 